\title{
APLICAÇÃO DE TESTES DE NORMALIDADE EM PUBLICAÇÕES NACIONAIS: LEVANTAMENTO BIBLIOGRÁFICO
}

Aryane Flauzino Machado, Aline Castilho de Almeida, Amanda Costa Araújo, Deisi Ferrari, Ítalo Ribeiro Lemes, Nathálie Clara Souto Faria, Thais de Sousa Lima, Rômulo Araújo Fernandes

Universidade Estadual Paulista, FCT, Presidente Prudente, SP. e-mail: aryfmachado@yahoo.com.br.

\section{RESUMO}

O objetivo do presente estudo foi investigar e identificar os artigos publicados em periódicos nacionais que aplicaram testes de normalidade, quais os mais utilizados, se há relação com o Qualis e se no decorrer dos anos há um aumento na utilização desses testes. Para elaboração da pesquisa foram escolhidos três periódicos nacionais indexados no SciELO. Foram analisadas publicações entre 2008 e 2012. A análise descritiva foi realizada no Excel 2007 e o teste de normalidade (Shapiro Wilk) pelo SPSS, assim como a comparação entre grupos (ANOVA one way), com nível de significância de $5 \%$. Dentre a totalidade dos Artigos Originais ( $n=871)$, foi observado que 358 citaram a utilização de teste de normalidade. Em suma, pode-se afirmar que a Revista Brasileira de Fisioterapia é responsável por $46 \%$ dessas publicações. A Revista Fisioterapia e Pesquisa e Revista Fisioterapia em Movimento são responsáveis por 30,2\% e 23,7\% das publicações, respectivamente. Os testes estatísticos mais utilizados foram Shapiro-Wilk ( $\mathrm{n}=163$ ) e Komolgorov-Smirnov ( $n=137$ ). Estes foram responsáveis por $83,8 \%$ das publicações que citaram testar a normalidade de seus dados. Ainda, 2,8\% executaram outros testes e 13,4\% não especificaram qual teste utilizado, porém, afirmaram testar a normalidade. Conclui-se então que o periódico nacional de maior Qualis, a Revista Brasileira de Fisioterapia, possui maior rigor criterioso em relação à análise estatística e que não há diferença estatisticamente significante no número de artigos publicados que aplicaram o teste de normalidade no decorrer dos anos. Observa-se também que o teste de Shapiro-Wilk foi o mais utilizado nas publicações.

Palavras-chave: estatística, dados numéricos, análise estatística, teste de normalidade.

\section{APPLICATION OF NORMALITY TESTS IN NATIONAL PUBLICATIONS: SURVEY BIBLIOGRAPHIC}

\begin{abstract}
The aim of this study was to investigate and identify the articles published in national journals that applied normality tests, which are the most used, if there is any relation with the Qualis and there over the years an increase in the use of these tests. For doing this research three national journals indexed in SciELO were chosen. Publications between 2008 and 2012 were analyzed. A descriptive analysis was performed in Excel 2007 and normality test (Shapiro Wilk) by SPSS, as well as the comparison between groups (ANOVA one-way), with significance level of $5 \%$. Among all of the original articles $(n=871)$, it was observed that 358 mentioned the use of normality test. Thus, it can be stated that the Brazilian Journal of Physical Therapy is responsible for $46 \%$ of these publications. Physical Therapy and Research and Physical Therapy in Movement account for 30.2\% and $23.7 \%$ of publications, respectively. Statistical tests were most used Shapiro-Wilk $(n=163)$ and Komolgorov-Smirnov $(n=137)$. These accounted for $83.8 \%$ of the publications cited test the normality of your data. Even more, other tests performed $2.8 \%$ and $13.4 \%$ did not specify which test is used, however, said test normality. It is concluded that the national journal of higher Qualis, Brazilian Journal of Physical Therapy, has more strict criteria in relation to statistical analysis and
\end{abstract}


no statistically significant difference in the number of published papers that applied the test of normality over the years. It is also observed that the Shapiro-Wilk test was used more often in publications.

Keywords: Statistics, numerical data, statistical analysis, Normality Tests. 


\section{INTRODUÇÃO}

A relação entre a atuação clínica e o ambiente científico tem sido substancialmente discutida em suas diferentes vertentes. A prática clínica baseada em evidências integra a experiência vivenciada na prática com evidências científicas de qualidade presentes na literatura ${ }^{1}$. Nesse sentido, a fisioterapia tem buscado participar de forma ativa na busca por evidências, o que pode ser justificado pelo aumento do volume e da acessibilidade da pesquisa clínica de alta qualidade nos últimos anos ${ }^{2}$.

A produção científica na fisioterapia tem apresentado tendência de crescimento, sendo pareada com o número de publicações nas demais áreas. Esses estudos são direcionados a áreas específicas, buscando publicações em periódicos de qualidade editorial, destacados por meio de bases de dados renomadas. Para que haja melhor qualidade, as publicações passam por processo criterioso de avaliação para então serem indexadas em bases bibliográficas ${ }^{1}$. Contudo, os crivos de avaliação não apresentam ainda padrões de excelência e, assim, os estudos brasileiros têm sido considerados incompletos frente a estudos internacionais, os quais têm contestado principalmente a metodologia utilizada ${ }^{3-5}$.
Nesse sentido, a ciências da saúde, de modo geral, tem incorporado em seus estudos, técnicas estatísticas como estratégia metodológica para a investigação científica a fim de apoiar inferências clínicas. A utilização de testes estatísticos mais complexos tem aumentado com o decorrer do tempo ${ }^{6,7}$, porém deve-se haver uma atenção especial na forma de utilização desses.

É importante verificar, antes das análises estatísticas, que qualquer uma das hipóteses incorridas em testes individuais não seja violada. A suposição de que a amostra utilizada no estudo é normalmente distribuída, pode acarretar grandes erros estatísticos. Muitas vezes autores assumem a normalidade sem a realização de testes para verificação, conforme sua conveniência. Quando adotado esse procedimento, a interpretação, compreensão e inferência dos dados tornam-se inválidas ${ }^{8}$.

A distribuição de dados, quando normal, apresenta uma curva de sino simétrica, sendo caracterizada por maior frequência central (no meio) e baixas frequências em seus extremos ${ }^{9}$. A normalidade dos dados é determinante nos métodos estatísticos paramétricos, além de permitir melhor compreensão para geração de dados $^{10}$.

Alguns testes estatísticos têm se destacado para a verificação de normalidade, 
entre eles estão o teste de KolmogorovSmirvov ${ }^{11,12}$ e o teste de Shapiro-Wilk. A utilização desses testes deve-se à simplicidade e disponibilidade na grande parte dos softwares estatísticos, como o SPSS, STATA e Minitab ${ }^{13}$.

Conforme exposto, a utilização de testes estatísticos para inferir sobre a normalidade da distribuição dos dados tornase uma ferramenta essencial para a validação dos dados ${ }^{14}$. Dessa forma, espera-se que revistas de maior Qualis devam ser mais criteriosas na avaliação de artigos para publicação em relação à suas análises estatísticas, apresentando maior número de artigos que realizam teste de normalidade em seus dados, assim como por meio de investigação no decorrer dos anos, espera-se que os artigos publicados apresentem uma tendência crescente em acrescentar tais testes em suas análises estatísticas.
O objetivo do presente estudo foi investigar e identificar os artigos publicados em periódicos nacionais que aplicaram testes de normalidade, quais os mais utilizados, se há relação com o Qualis e se no decorrer dos anos há aumento na utilização desses testes.

\section{METODOLOGIA}

Seleção dos periódicos

Para elaboração da pesquisa foram escolhidos três periódicos de importante revelância no cenário da pesquisa em Fisioterapia no Brasil e indexadas no SciELO: Revista Brasileira de Fisioterapia, Revista Fisioterapia em Movimento e Revista Fisioterapia e Pesquisa, descritas por seus Qualis e Fatores de Impacto no Quadro 1.

Quadro 1. Descrição dos periódicos analisados no ano de 2012.

\begin{tabular}{cccc}
\hline Periódicos & ISSN & Qualis - Área 21 & Fator de Impacto \\
\hline $\begin{array}{c}\text { Revista Brasileira } \\
\text { de Fisioterapia }\end{array}$ & $1413-3555$ & A2 & 1,00 \\
\hline $\begin{array}{c}\text { Revista Fisioterapia } \\
\text { em Movimento }\end{array}$ & $0103-5150$ & B1 & 0,29 \\
\hline $\begin{array}{c}\text { Revista Fisioterapia } \\
\text { e Pesquisa }\end{array}$ & $1809-2950$ & B1 & 0,19 \\
\hline
\end{tabular}

Extração dos dados

A extração dos dados foi realizada por sete avaliadores treinados, os quais realizaram a classificação dos artigos, dividindo-os em Artigos Originais e Outras Categorias. 
Classificados, os avaliadores extraíram dos Artigos Originais informações relacionadas à Análise Estatística, selecionando os que utilizaram Teste de Normalidade para sua análise. A partir de então, foram identificados os Testes de Normalidade mais utilizados na preparação de artigos.

Foram analisadas publicações dos três periódicos dos anos de 2008 a 2012.

\section{Análise estatística}

A análise descritiva dos dados foi realizada no software Microsoft Office Excel 2007. Do pacote estatístico Statistical Package for the Science (SPSS - version 18, Chicago, IL, USA) foi utilizado o teste ShapiroWilk para testar a normalidade dos dados e o teste de ANOVA (one way) para comparação entre os grupos. Foi adotado o nível de $5 \%$ de significância.

\section{RESULTADOS}

Dos 871 artigos originais encontrados, 324 foram publicados na Revista Brasileira de Fisioterapia, 290 na Revista Fisioterapia em Movimento e 257 na Revista Fisioterapia e Pesquisa. Dentre a totalidade dos artigos Originais $(n=871)$, foi observado que apenas 358 citaram utilizar algum teste de normalidade para verificar seus dados, sendo 165 na Revista Brasileira de Fisioterapia, 108 na Revista Fisioterapia e Pesquisa e 85 da Revista Fisioterapia em Movimento, descritos no Fluxograma 1.

Em suma, pode-se afirmar que a Revista Brasileira de Fisioterapia é responsável por $46 \%$ das publicações que utilizaram teste de análise de normalidade. A Revista Fisioterapia e Pesquisa e Revista Fisioterapia em Movimento são responsáveis por $30,2 \%$ e $23,7 \%$ das publicações, respectivamente. 


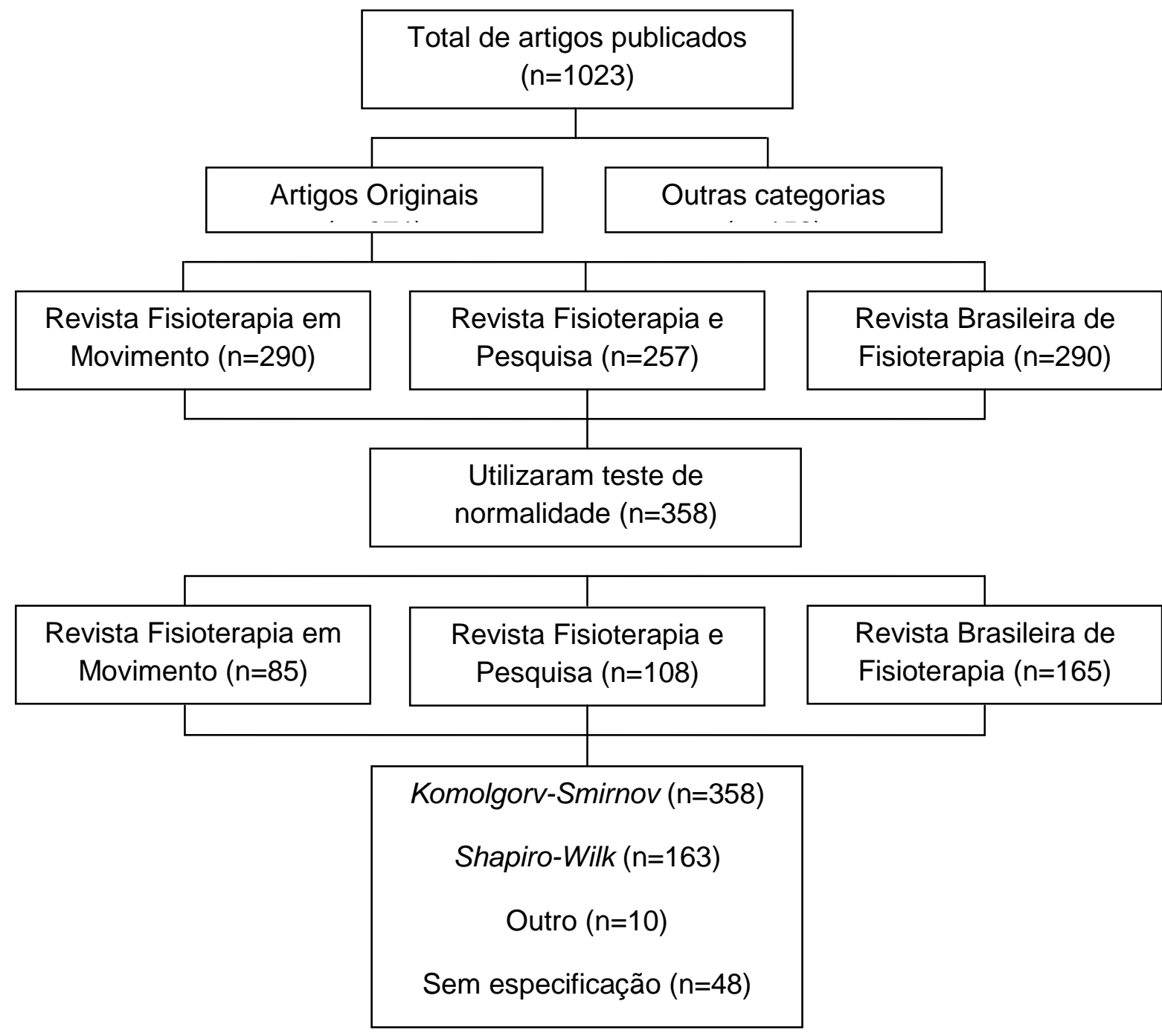

Os testes estatísticos mais utilizados nas publicações analisadas para testar a normalidade foram Shapiro-Wilk $(n=163)$ e Komolgorov-Smirnov $(n=137)$. Estes foram responsáveis por $83,8 \%$ das publicações que citaram testar a normalidade de seus dados. Ainda, 2,8\% executaram outros testes e 13,4\% não especificaram qual teste utilizado, porém, afirmaram testar a normalidade.

Conforme mostra a Tabela 1, o estudo revela que apenas $29 \%$ dos artigos publicados na Revista Fisioterapia em
Movimento realizaram algum tipo de teste de normalidade. Analisando os artigos publicados na Revista Fisioterapia e Pesquisa apenas $42,8 \%$ mencionaram a utilização de algum teste de normalidade em seus dados. Quanto à Revista Brasileira de Fisioterapia $50,3 \%$ dos artigos avaliados citaram submeter seus dados a testes de normalidade. 
Tabela 1. Descrição de artigos originais que utilizaram teste de normalidade.

\begin{tabular}{ccccccccccc}
\hline & \multicolumn{1}{c}{ Fisioterapia em Movimento } & \multicolumn{3}{c}{ Fisioterapia e Pesquisa } & \multicolumn{3}{c}{ Brasileira de Fisioterapia } \\
\cline { 2 - 10 } & $\begin{array}{c}\text { Ano } \\
\text { original }\end{array}$ & N teste & $\%$ & $\begin{array}{c}\text { N } \\
\text { original }\end{array}$ & $\begin{array}{c}\text { N } \\
\text { teste }\end{array}$ & $\%$ & $\begin{array}{c}\text { N } \\
\text { original }\end{array}$ & $\begin{array}{c}\text { N } \\
\text { teste }\end{array}$ & $\%$ \\
\hline 2008 & 43 & 11 & 25,6 & 48 & 11 & 22,9 & 65 & 27 & 41,5 \\
2009 & 44 & 14 & 31,8 & 51 & 16 & 31,4 & 63 & 24 & 38,1 \\
2010 & 49 & 10 & 20,4 & 51 & 20 & 39,2 & 65 & 37 & 56,9 \\
2011 & 77 & 22 & 28,6 & 54 & 28 & 51,9 & 66 & 38 & 57,6 \\
2012 & 77 & 27 & 35,1 & 53 & 35 & 66,0 & 65 & 37 & 56,9 \\
\hline Total & 290 & $84 *$ & 29,0 & 257 & 110 & 42,8 & 324 & $163 *$ & 50,3 \\
\hline
\end{tabular}

* p<0,05 na comparação entre as Revistas Fisioterapia em Movimento e Brasileira de Fisioterapia.

$\mathrm{N}$ original - Número de artigos publicados caracterizados como original.

$\mathrm{N}$ teste - Número de artigos publicados que aplicaram teste de normalidade.

A proporção de publicações que utilizaram ferramentas para testar as normalidades dos dados é representada na Figura 1. A Revista Fisioterapia em Movimento apresentou queda na utilização desses testes no ano de 2010, gerando a partir desse ponto um trajeto crescente. A Revista Fisioterapia e Pesquisa apresentou ao longo dos cinco anos analisados, curva crescente, representando em 2012 a revista mais criteriosa nesse quesito. Já a Revista Brasileira de Fisioterapia parte desde 2008 com a maior proporção, porém no período entre 2010 e 2012 há estabilidade na proporção de publicações que utilizam testes de normalidade, sendo em 2012 ultrapassada pela Revista Fisioterapia e Pesquisa. 


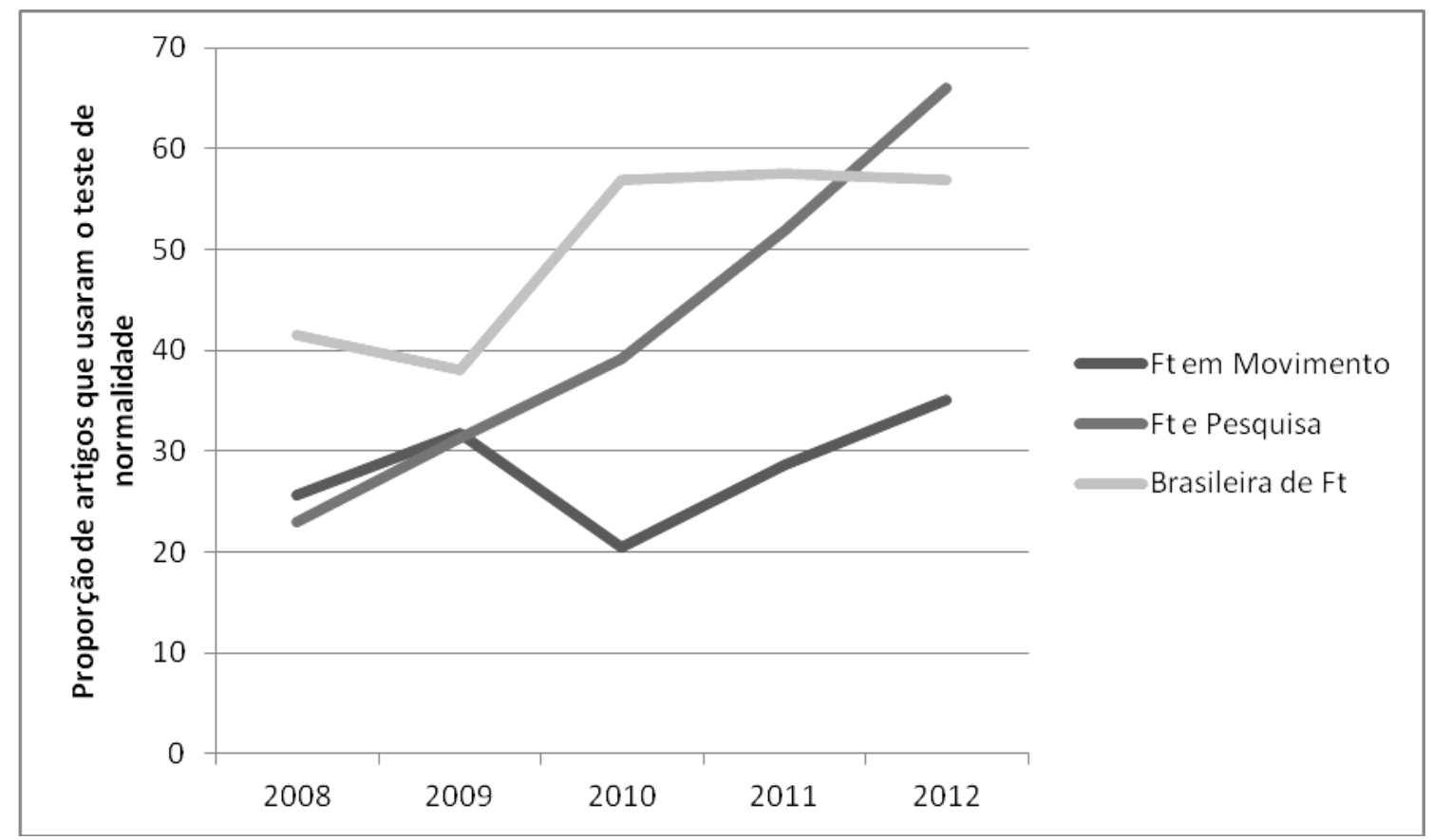

Figura 1. Proporção de artigos que usaram o teste de normalidade por ano.

\section{DISCUSSÃO}

A partir dos achados do presente estudo, pode-se observar que a Revista Brasileira de Fisioterapia possui maior número de artigos publicados que realizam o teste de normalidade em seus dados, o que corrobora com a hipótese inicial, na qual a revista de maior Qualis seria mais criteriosa para avaliação dos artigos em relação à suas análises estatísticas.

Nota-se ainda que houve diferença estatisticamente significante no número de artigos publicados nessa revista que realizaram o teste de normalidade em comparação com a revista Fisioterapia e Movimento. Entretanto, não foram observadas diferenças estatisticamente significantes no número de artigos publicados com tais características no decorrer dos anos. Conforme foi inicialmente hipotetizado, observou-se apenas tendência de aumento dessa proporção, principalmente para a Revista Fisioterapia e Pesquisa, como observado na Figura 1.

As atuações exercidas em âmbito prático, ou seja, atuações clínicas são norteadas pelas evidências científicas. Dessa forma, falhas metodológicas, tanto de desenvolvimento da pesquisa quanto de análise dos dados obtidos, podem levar clínicos a uma interpretação errônea do estudo e de seus achados. É de extrema importância que a metodologia utilizada, principalmente em relação à análise estatística, seja correta e precisa.

Afshar et al. $^{16}$ apontaram, em levantamento semelhante, que $40 \%$ dos estudos por eles analisados, apresentaram 
análises estatística incorretas. Em se tratando de artigos originais, a aplicação de testes de normalidade é imprescindível. Neste estudo, $41 \%$ dos artigos analisados usaram teste de normalidade, o que parece coerente com publicações internacionais.

Orso et al. ${ }^{17}$, apesar de constituírem a área de cirurgia do aparelho digestivo, encontraram em levantamento de 20 anos, uma crescente em relação ao uso da estatística analítica. Porém, ainda existe a necessidade da correta aplicação dos procedimentos estatísticos.

\section{CONCLUSÃO}

Conclui-se então que, dentre os periódicos nacionais analisados, o de maior Qualis, a Revista Brasileira de Fisioterapia, possui maior rigor criterioso em relação à análise estatística e que não há diferença estatisticamente significante no número de artigos publicados que aplicaram o teste de normalidade no decorrer dos anos. Observase também que o teste de Shapiro-Wilk foi o mais utilizado nas publicações.

\section{CONFLITO DE INTERESSE}

Os autores declaram não haver qualquer potencial conflito de interesse que possa interferir na imparcialidade deste trabalho científico.

\section{REFERÊNCIAS}

1. Coury HJCG, Vilella I. Perfil do pesquisador fisioterapeuta brasileiro. Rev Bras Fisiot. 2009;13(4):356-363. DOI: http://dx.doi.org/10.1590/S1413$\underline{35552009005000048}$

2. Maher CG, Sherrington C, Elkins $M$, Herbert RD, Moseley AM. Challenges for evidence-based physical therapy: accessing and interpreting high-quality evidence on therapy. Phys Ther. 2004;84(7):644-654.

3. Warden SJ. Letter to the editor. On "Journal publication productivity..." Richter et al. Phys Ther. 2008;88(3):376386.

4. Maher C. Letter to the editor. On "Journal publication productivity..." Richter et al. Phys Ther. 2008;88(4):538-539.

5. Harris SR. Letter to the editor. On "Journal publication productivity..." Richter et al. Phys Ther. 2008;88(6):791. http://dx.doi.org/10.2522/ptj.2008.88.6.7 $\underline{91}$

6. Pereira JCR. Tamanho de amostra: uma necessidade real ou um capricho cultural? Arteríola. 2002;4(1):13-16.

7. Orso IRB, Pereira JCR, D'Albuquerque LAC, Cecconello I, Jukemura J. Critical analysis of the use of statistical tests in Brazilian publications related to digestive tract surgery. Rev Assoc Médica Bras. 2011;57(1):35-41. DOI: http://dx.doi.org/10.1590/S010442302011000100013

8. Ahad NA, Yin TS, Othman AR, Yaacob CR. Sensitivity of Normality Tests to Nonnormal Data. Sains Malaysiana. 2011;40(6):637-641. 
9. Gravetter FJ, Wallnau LB. Statistics for the behavioral sciences. Wadsworth: Thomson Learning; 2000.

10. Chambers JM. Graphical methods for data analysis. New York: Champman \& Hall; 1983.

11. Kolmogorov AN. Sulla determinazione empirica di una legge di distribuzione, Giornale dell' Instituto Italiano degli Attuari. 1993;4:83-91. www.citeulike.org/user/mendonca/article $\lcm{1213059}$

12. Smirnov NV. Sui la distribution de $\mathbf{w}^{2}$ (Criterium de M.R.v. Mises), Comptes Rendus. 1936;449-452.

13. Shapiro SS, Wilk MB. An analysis of variance test for normality (complete samples). Biometi-ika. 1965;52(3 and 4):591.

14. Pereira Filho NA, Ferreira DF. Teste monte carlo de normalidade univariado baseado em distancias. Rev Bras Biometria. 2012;30(3):401-416.

15. Normando D, Tjäderhane L, Quintão CCA. A escolha do teste estatístico - um tutorial em forma de apresentação em PowerPoint. Dental Press J Orthod. 2010;15(1):101-106.

16. Afshar K, Jafari S, Seth A, Lee JK, MacNeily AE. Publications by the American Academy of Pediatrics Section on Urology: The Quality of Research Design and Statistical Methodology. J Urol. 2009;182(4 Suppl):1906-1910. DOI: http://dx.doi.org/10.1016/j.juro.2009.02.0 $\underline{66}$

17. OIR Bonotto, JCR Pereira, LAC D'Albuquerque, I Cecconello, J Jukemura. Critical analysis of the use of statistical tests in Brazilian publications related to digestive tract surgery. Rev Assoc Médica
Bras. 2011;57(1):35-41.

http://dx.doi.org/10.1590/S0104$\underline{42302011000100013}$

Recebido para publicação em 10/04/2014

Revisado em 08/12/2014

Aceito em 18/03/2015 